\title{
Comparison of Sclerostin Levels in Gingival Crevicular Fluid before and after Nonsurgical Therapy in Smokers and Nonsmokers with Chronic Periodontitis
}

\author{
Gowtham Krishna ${ }^{1}$, Saravana K Ravindran ${ }^{2}$, Pratebha Balu ${ }^{3}$, Jananni Muthu ${ }^{4}$, Sakthidevi Sathiyaseelan ${ }^{5}$
}

\begin{abstract}
Aim and objective: This study aims to assess gingival crevicular fluid levels of sclerostin before and after nonsurgical periodontal therapy in smokers and nonsmokers with chronic generalized periodontitis.

Materials and methods: Subjects with generalized chronic periodontitis were divided into two groups: Group I-nonsmokers with chronic periodontitis $(n=18)$ and group II-smokers with chronic periodontitis $(n=18)$. Baseline clinical parameters were recorded and a GCF sample was collected for assessment of sclerostin levels. All the patients received nonsurgical periodontal therapy. GCF sampling and clinical periodontal measurements were repeated 4 weeks after completion of SRP. Sclerostin levels were analyzed using an enzyme-linked immunosorbent assay (HUMAN SOST ELISA KIT). The data were tested statistically by the Student's $t$-test: Intragroup analysis was done using paired $t$-test and intergroup was done using independent $t$-test.

Results: Probing pocket depth and clinical attachment loss were decreased significantly in both smokers and nonsmokers after nonsurgical therapy. The levels of sclerostin were higher in the smoker group when compared with nonsmokers. The mean sclerostin levels in smokers and nonsmokers at baseline were $354 \pm 141$ and $164.87 \pm 59.8 \mathrm{pg} / \mathrm{mL}$, respectively. There is a significant decrease in sclerostin levels from (164-136 $\mathrm{pg} / \mathrm{mL})$ in nonsmokers after nonsurgical therapy $(p=0.001)$.

Conclusion: Sclerostin levels were significantly higher in smokers when compared with nonsmokers at the baseline and exhibited persistent higher levels even after periodontal therapy in smokers.

Clinical significance: Sclerostin is an osteocyte secreted soluble antagonist of the wnt/ $\beta$ catenin signaling pathway which is a requisite for osteoblast development and osteogenesis. Increased sclerostin levels in smokers increase and hasten periodontal destruction. Therefore, sclerostin can be a promising therapeutic target for control of osteoclastic activity and promotion of osteogenesis in chronic periodontitis and more so in smokers.

Keywords: Chronic periodontitis, GCF, Nonsurgical periodontal therapy, Observational study, Sclerostin, Smoking.

World Journal of Dentistry (2021): 10.5005/jp-journals-10015-1853
\end{abstract}

\section{INTRODUCTION}

Chronic periodontitis is a multifactorial inflammatory disease of the gingival tissues of susceptible individuals in response to bacterial plaque accumulation. The onset of this disease is initiated by microorganisms and progression. ${ }^{1}$ Bone destruction driven by the inflammatory process involves a "two-way" interaction between the inflammatory infiltrate and cells responsible for bone homeostasis. Exacerbation in the production of these inflammatory mediators results in soft tissue destruction and activation of the bone resorption mechanism. ${ }^{2}$

The regulation of bone metabolism is a complex process involving diverse signal transduction pathways both catabolic (RANK-RANKL) and anabolic (Wnt signaling) pathways. ${ }^{3}$ Regulation of Wnt signaling is mediated through post-translational modification of Wnts, receptor regulation, and antagonist binding. Sclerostin and dickkopf-1 have been identified as being endogenous antagonists of the Wnt pathway. ${ }^{4}$

Sclerostin, a product of the SOST gene, is a secreted glycoprotein that binds low-density lipoprotein receptor-related protein 5 and blocks the Wnt signaling pathway. It is secreted by cementocytes and osteocytes. Its expression, which suppresses osteoclastogenesis and reduces the viability of osteoblasts and osteocytes, leads to unbalanced bone turnover in favor of bone \begin{tabular}{l}
\hline${ }^{1-5}$ Department of Periodontology, Indira Gandhi Institute of Dental \\
Sciences, Sri Balaji Vidyapeeth, Puducherry, India
\end{tabular} Corresponding Author: Gowtham Krishna, Department of Periodontology, Indira Gandhi Institute of Dental Sciences, Sri Balaji Vidyapeeth, Puducherry, India, Phone: +91 7358662884, e-mail: drgowtham167@gmail.com

How to cite this article: Krishna G, Ravindran SK, Balu P, et al. Comparison of Sclerostin Levels in Gingival Crevicular Fluid before and after Nonsurgical Therapy in Smokers and Nonsmokers with Chronic Periodontitis. World J Dent 2021;12(5):363-366.

Source of support: Nil

Conflict of interest: None

resorption. Considering the role of sclerostin in bone destruction, it can be speculated that this protein can influence bone destruction in periodontal diseases as well and can be a potential biomarker to assess bone destruction in periodontal diseases. ${ }^{5}$

RANK-RANKL-mediated bone destruction in periodontal diseases has been widely researched and reported. However, the endogenous antagonists like sclerostin and dickkopf proteins, of the Wnt signaling pathway have not been explored much. Only a 
few studies have assessed the anabolic pathway, by measuring the sclerostin levels in periodontal health and disease.

Studies have reported an increase in sclerostin levels in periodontitis patients as compared to healthy subjects. ${ }^{6}$ Reduction in levels of sclerostin after nonsurgical therapy in chronic periodontitis patients has also been reported. ${ }^{7}$ Similarly, in diabetic patients with chronic periodontitis and habit of smoking, a marked upregulation of SOST was observed. ${ }^{8}$

Smoking is a well-established risk factor for periodontitis, and the components of tobacco especially nicotine metabolites suppress osteoblasts proliferation and stimulate alkaline phosphatase activity. ${ }^{9}$

Numerous studies have assessed RANK/RANKL-mediated alveolar bone destruction, but to the best of our knowledge, no study has assessed the influence of smoking-mediated catabolic bone destruction in periodontal disease.

In lieu of the above the aim of the present study to compare and evaluate the gingival crevicular fluid levels of sclerostin before and after nonsurgical therapy in smokers and nonsmokers with chronic periodontitis.

\section{Materials and Methods}

The present study was carried out in the Department of Periodontology, Indira Gandhi Institute of Dental Sciences (IGIDS), Sri Balaji Vidyapeeth (Deemed to be University) from August 2019 to March 2020. This study was approved by Institutional Human Ethics Committee, IGIDS (IGIDSIEC2019NRP04PGGOPAI). Written informed consent was obtained from all patients before their enrolment in the study.

\section{Sample Size Calculation}

The sample size was calculated using standardized statistical software G Power 3.0. Considering $2.18 \pm 4.16$ and $5.87 \pm 4.83$ as mean and standard deviation from the previous study by Nurkan et al. (2009), using the formula

$$
n \geq \frac{2\left(Z_{1-\beta}+Z_{1-\alpha / 2}\right)^{2}}{(\delta \text { difference } / \alpha \text { difference })^{2}}+Z_{1-\alpha / 2} / 2
$$

Sample size needed in each group is calculated as 18.

\section{Study Participants}

Patients diagnosed with generalized moderate/severe chronic periodontitis as per the American Academy of Periodontology (AAP) criteria 1999 were included in the study. Participants had at least two teeth with probing pocket depth $\geq 5 \mathrm{~mm}$ and clinical attachment loss $\geq 4 \mathrm{~mm}$ with bleeding on probing at the affected sites in $>30 \%$ of sites were selected. Smokers were participants who had smoked 100 or more cigarettes over their lifetime and smoked at the time of the examination and nonsmokers were participants who have not smoked $\geq 100$ cigarettes in their lifetime and do not currently smoke (according to CDC). Participants were excluded from the study if they exhibited either presence of uncontrolled systemic disease, pregnancy, history of bone remodeling drug therapy, periodontal therapy during the past 6 months, former smokers and antibiotics, etc.

Subjects with chronic periodontitis based on their smoking status were divided into two groups: Group I comprises nonsmokers with chronic periodontitis $(n=18)$ and group II comprises smokers with chronic periodontitis $(n=18)$.

In both the groups, the clinical periodontal parameters (probing pocket depth and clinical attachment level) were recorded at baseline, following this GCF sample was collected from the deepest pocket for evaluation of sclerostin levels. Nonsurgical periodontal therapy comprising of scaling and root planing was done on the same appointment and oral hygiene instructions were given. All patients were recalled at 1 month after nonsurgical periodontal therapy for the follow-up. One month postoperatively, the same clinical periodontal parameters were recorded and GCF sample was collected from the same site. A total of 36 patients finished the 1 month follow-up period; there is no dropout of cases in this study. Recording of clinical periodontal parameters, nonsurgical periodontal therapy, and GCF sample collection in all cases was performed by the principal investigator.

Clinical parameters were recorded using the UNC-15 probe. Scaling was done ultrasonic scaler $\left(\mathrm{EMS}^{\mathrm{TM}}\right)$ and root planing was done using and Gracey area-specific curettes.

\section{GCF Sample Collection}

GCF samples were collected by intracrevicular method as described by Mann et al. in 1963 using grade I Whatman filter paper. Samples were collected from sites with the deepest pocket depth. Samples were taken from the same sites at baseline and 1-month postnonsurgical therapy in smokers and nonsmokers with chronic periodontitis. The selected teeth were isolated with cotton rolls and air-dried. A supragingival plaque was removed carefully with a sterile scaler. Paper strips were held using a tweezer and it was placed into the gingival crevice until mild resistance was felt and were left in position for 30 seconds. Strips contaminated with saliva or blood were discarded. Two strips from each patient were pooled to make it as one sample which was then transferred to a sterile Eppendorf tube $(1.5 \mathrm{~mL})$ containing $250 \mu \mathrm{L}$ phosphate-buffered saline. All samples were immediately stored at $-80^{\circ} \mathrm{C}$ until the day of laboratory analysis. Sclerostin levels were analyzed using an enzyme-linked immunosorbent assay (HUMAN SOST ELISA KIT).

\section{Statistical Analysis}

The results of all parameters (PPD, CAL, and SOST) are presented as mean and standard deviation. Inferential analysis for intragroup was done using paired $t$-test and intergroup has been done using independent $t$-test.

\section{Results and Observations}

The mean probing depth in group I (nonsmokers with periodontitis) at baseline and at 1 month postoperatively was $3.83 \pm 0.66$ and 3.49 $\pm 0.67 \mathrm{~mm}$. The mean clinical attachment level at baseline and at 1 month was $2.27 \pm 0.76$ and $2.10 \pm 0.77 \mathrm{~mm}$, respectively (Table 1).

The mean probing depth in group II (smokers with periodontitis) for smokers with periodontitis at baseline and at 1 month was 3.98 \pm 0.73 and $3.72 \pm 0.74 \mathrm{~mm}$. The mean clinical attachment level at baseline and at 1 month was $2.78 \pm 1.2$ and $2.63 \pm 1.2 \mathrm{~mm}$, respectively (Table 2 ).

The mean probing depth in nonsmokers and smokers at baseline was $3.83 \pm 0.66$ and $3.98 \pm 0.73 \mathrm{~mm}$. The mean probing depth in nonsmokers and smokers at 1 month was $3.49 \pm 0.67$ and $3.72 \pm 0.74 \mathrm{~mm}$, respectively. The mean clinical attachment level in nonsmokers and smokers at baseline was $2.27 \pm 0.76$ and 
$2.78 \pm 1.2 \mathrm{~mm}$. The mean clinical attachment level in nonsmokers and smokers at 1 month was $2.10 \pm 0.77$ and $2.63 \pm 1.2 \mathrm{~mm}$, respectively (Table 3 ).

The mean sclerostin levels in nonsmokers at baseline and at 1 month were $164.87 \pm 59.8$ and $136 \pm 45.2 \mathrm{pg} / \mathrm{mL}$. Group II: The mean sclerostin levels in smokers at baseline and at 1 month were $335 \pm 141$ and $328 \pm 148 \mathrm{pg} / \mathrm{mL}$, respectively.

The mean sclerostin levels in smokers and nonsmokers at baseline were $335 \pm 141$ and $164.87 \pm 59.8 \mathrm{pg} / \mathrm{mL}$, respectively. The mean sclerostin levels in smokers and nonsmokers at 1-month postoperatively were $136 \pm 45.2$ and $328 \pm 148 \mathrm{pg} / \mathrm{mL}$, respectively.

There is a significant decrease in sclerostin levels in nonsmokers from baseline to 1 month were significant $(p=0.001)$. Whereas the decrease in sclerostin levels in smokers from baseline to 1 month were not statistically significant $(p=0.843)$ (Fig. 1).

\section{Discussion}

The present study included patients with generalized chronic periodontitis. Subjects were divided into two groups: Group I-nonsmokers with chronic periodontitis $(n=18)$ and group IIsmokers with chronic periodontitis $(n=18)$. All patients received nonsurgical periodontal therapy (scaling and root planing). GCF sampling for sclerostin and clinical periodontal parameters (probing pocket depth and clinical attachment level) were assessed at baseline and 1 month after nonsurgical periodontal therapy.

Both group I and II exhibited a significant reduction in PPD and CAL at 1-month postoperatively. Comparing clinical outcomes

Table 1: Comparison of parameters at baseline and 1 month in group I (nonsmokers)

\begin{tabular}{lrlll}
\hline Parameters & \multicolumn{1}{c}{ Mean \pm SD } & Tvalue & Df & p value \\
\hline PPD_BASELINE & $3.83 \pm 0.66$ & 9.332 & 17 & $0.000^{*}$ \\
PPD_POST $(\mathrm{mm})$ & $3.49 \pm 0.67$ & & & \\
CAL_BASELINE & $2.27 \pm 0.76$ & 8.841 & 17 & $0.000^{*}$ \\
CAL_POST $(\mathrm{mm})$ & $2.10 \pm 0.77$ & & & \\
SOST_BASELINE & $164.87 \pm 59.8$ & 3.377 & 17 & $0.004^{*}$ \\
SOST_POST $(\mathrm{pg} / \mathrm{mL})$ & $136.0 \pm 45.2$ & & & \\
\hline
\end{tabular}

*Statistically significant as $p<0.005$

Table 2: Comparison of parameters at baseline and 1 month in group II (smokers)

\begin{tabular}{lllll}
\hline Parameters & Mean $\pm S D$ & Tvalue & Df & p value \\
\hline PPD_BASELINE & $3.98 \pm 0.73$ & 10.004 & 14 & $0.000^{*}$ \\
PPD_POST $(\mathrm{mm})$ & $3.72 \pm 0.74$ & & & \\
CAL_BASELINE & $2.78 \pm 1.2$ & 6.831 & 14 & $0.000^{*}$ \\
CAL_POST $(\mathrm{mm})$ & $2.63 \pm 1.2$ & & & \\
SOST_BASELINE & $335 \pm 141$ & 0.202 & 14 & 0.843 \\
SOST_POST $(\mathrm{pg} / \mathrm{mL})$ & $328 \pm 148$ & & & \\
\hline
\end{tabular}

*Statistically significant as $p<0.005$ between the groups, nonsmokers with periodontitis had a greater reduction in PPD compared with smokers with periodontitis.

The clinical and microbiological effects of nonsurgical periodontal therapy in smokers and nonsmokers were compared by Darby et al., he reported that 2 months following therapy, probing depth reduction was higher in nonsmokers when compared with smokers. ${ }^{10}$ Farina et al., investigated the changes in probing pocket depth 1 month following nonsurgical periodontal treatment and found that nonsmokers had a greater reduction in probing pocket depth 1 month postoperatively. ${ }^{11}$ Chang et al. in a systematic review reported that following nonsurgical therapy, smokers experienced less probing depth reduction when compared with nonsmokers. ${ }^{12}$

The difference in tissue response between smokers and nonsmokers is reportedly due to the difference in gingival blood flow and the number of blood vessels in the marginal gingiva. An increase in gingival volume due to the increase in blood vessels seems to be less evident in smokers. ${ }^{13}$ Differences in healing are also related to impaired function of fibroblasts and PMN phagocytosis in smoking patients. All these factors collectively affect the outcome of periodontal therapy in smoking patients. ${ }^{14}$

In the present study, a significant increase in levels of sclerostin was observed in smokers as compared to nonsmokers. Rezaei Esfahrood et al. compared the levels of sclerostin in chronic periodontitis with healthy subjects and reported that sclerostin levels were significantly higher in periodontitis patients than in healthy individuals. ${ }^{6}$ Chatzopoulos et al. related sclerostin (SOST), WNT-5a, and TNF-a levels between chronic periodontitis and periodontally healthy site and identified their significance as chronic periodontitis diagnostic markers. He stated that in the generalized moderate and severe chronic periodontitis subgroup, the protein levels of SOST were significantly higher compared

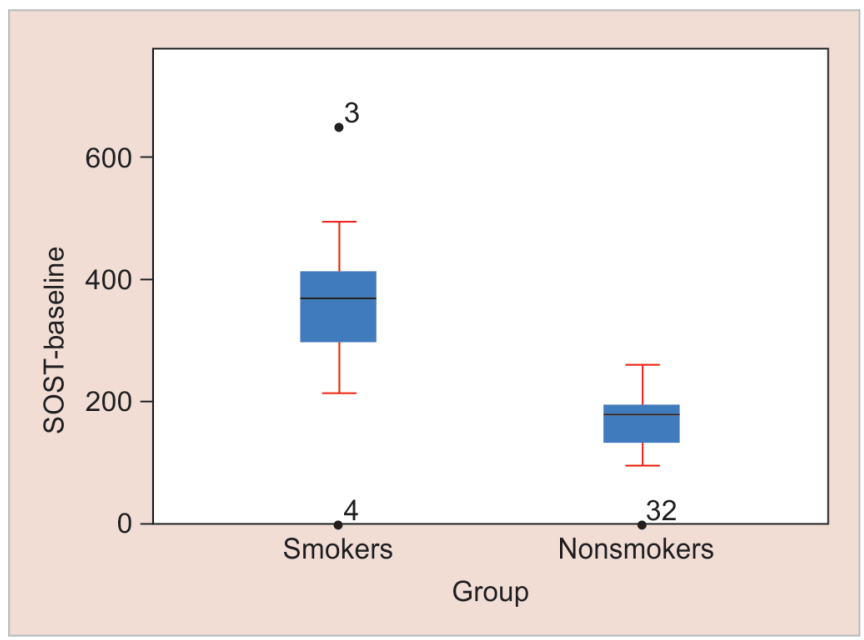

Fig. 1: Intergroup comparison of sclerostin levels at baseline

Table 3: Comparison of parameters between group I and group II

\begin{tabular}{|c|c|c|c|c|c|c|}
\hline & \multicolumn{2}{|c|}{$P P D(\mathrm{~mm})$} & \multicolumn{2}{|c|}{$C A L(m m)$} & \multicolumn{2}{|c|}{ SOST $(p g / m L)$} \\
\hline & Baseline & Post-op & Baseline & Post-op & Baseline & Post-op \\
\hline Group I & $3.83 \pm 0.66$ & $3.49 \pm 0.67$ & $2.27 \pm 0.76$ & $2.10 \pm 0.77$ & $164.87 \pm 59.8$ & $136.0 \pm 45.2$ \\
\hline Group II & $3.98 \pm 0.73$ & $3.72 \pm 0.74$ & $2.78 \pm 1.2$ & $2.63 \pm 1.2$ & $335 \pm 141$ & $328 \pm 148$ \\
\hline$p$ value & $0.000^{*}$ & $0.000^{*}$ & $0.000^{*}$ & $0.000^{*}$ & $0.000^{*}$ & $0.000^{*}$ \\
\hline
\end{tabular}

*Statistically significant as $p<0.005$ 
with the generalized healthy subgroup. ${ }^{15}$ Miranda et al. evaluated sclerostin (SOST) and Dickkopf (DKK)-1 levels and confirmed that SOST and Dickkopf were upregulated in chronic periodontitis patients with diabetes mellitus and/or smoking. ${ }^{8}$ García-Martín et al. assessed serum sclerostin in type 2 diabetes mellitus patients, and evaluated its relationship with bone metabolism, and concluded that type 2 diabetes mellitus patients had significantly higher levels of sclerostin than control subjects. ${ }^{16}$ Gingival tissues and serum from systemically stable patients diagnosed with chronic periodontitis and periodontal health were obtained in a cross-sectional analysis, and SOST mRNA and protein levels were measured. In the gingival tissue and serum samples of chronic periodontitis patients, the mRNA and protein levels of SOST were statistically significantly upregulated in comparison to periodontally healthy patients. ${ }^{17}$

From the previous studies, it can be inferred that sclerostin is associated with more bone destruction. In the present study, sclerostin levels in smokers were greater than nonsmokers indicating that smokers have more bone destruction than nonsmokers. Higher levels of sclerostin were also associated with peri-implantitis. Yakar et al. evaluated the gingival crevicular fluid and the peri-implant crevicular fluid level of sclerostin, TWEAK, RANKL, and OPG in periodontal and peri-implant tissues in disease and health conditions. He concluded that significantly higher levels of sclerostin in the peri-implantitis group than in the peri-implant health group. ${ }^{18}$

In the present study, sclerostin levels were measured in both the groups, 1 month postoperatively in the nonsmokers' group sclerostin levels reduced from baseline $(164.87 \pm 59.8 \mathrm{pg} / \mathrm{mL})$ to 1 month $(136.0 \pm 45.2 \mathrm{pg} / \mathrm{mL})$, and in the smokers' group, sclerostin levels reduced from baseline $(335 \pm 141 \mathrm{pg} / \mathrm{mL})$ to 1 month $(328 \pm$ $148 \mathrm{pg} / \mathrm{mL}$ ). The reduction in sclerostin levels following nonsurgical periodontal therapy was statistically significant $(p=0.001)$ in group I, whereas no statistically significant reduction was found in group II $(p=0.843)$. Balli et al. analyzed the changes in the levels and relative ratios of sclerostin, osteoprotegerin (OPG), and nuclear factor-B ligand-receptor activator (RANKL) in gingival crevicular fluid (GCF) of patients with periodontitis after nonsurgical periodontal therapy and concluded that sclerostin levels were significantly higher in patients with chronic periodontitis than in healthy people and reduced after periodontal nonsurgical therapy. ${ }^{7}$

\section{Conclusion}

Sclerostin levels were significantly higher in smokers when compared with nonsmokers at the baseline and exhibited persistent higher levels even after periodontal therapy in smokers, suggesting the role of sclerostin as prognostic biomarkers in periodontal disease.

\section{References}

1. Lamont RJ, Koo H, Hajishengallis $\mathrm{G}$. The oral microbiota: dynamic communities and host interactions. Nat Rev Microbiol 2018;16(12):745759. DOI: 10.1038/s41579-018-0089-x.
2. Graves DT, Oates T, Garlet GP. Review of osteoimmunology and the host response in endodontic and periodontal lesions. J Oral Microbiol 2011;3(1):5304. DOI: 10.3402/jom.v3i0.5304.

3. Phan TC, Xu J, Zheng MH. Interaction between osteoblast and osteoclast: impact in bone disease. Histol Histopathol 2004;19(4):1325-1344. DOI: 10.14670/HH-19.1325.

4. Kim JH, Liu X, Wang J, et al. Wnt signaling in bone formation and its therapeutic potential for bone diseases. Ther Adv Musculoskelet Dis 2013;5(1):13-31. DOI: 10.1177/1759720X12466608.

5. Baron R, Rawadi G. Targeting the Wnt/beta-catenin pathway to regulate bone formation in the adult skeleton. Endocrinology 2007;148(6):2635-2643. DOI: 10.1210/en.2007-0270.

6. Rezaei Esfahrood Z, Yadegari Z, Veysari SK, et al. Gingival crevicular fluid levels of sclerostin in chronic periodontitis and healthy subjects. J Korean Assoc Oral Maxillofac Surg 2018;44(6):289-292. DOI: 10.5125/ jkaoms.2018.44.6.289.

7. Balli U, Aydogdu A, Dede FO, et al. Gingival crevicular fluid levels of sclerostin, osteoprotegerin, and receptor activator of nuclear factor-KB ligand in periodontitis. J Periodontol 2015;86(12):1396-1404. DOI: 10.1902/jop.2015.150270.

8. Miranda TS, Napimoga MH, Feres M, et al. Antagonists of Wnt/ $\beta$ catenin signalling in the periodontitis associated with type 2 diabetes and smoking. J Clin Periodontol 2018;45(3):293-302. DOI: 10.1111/ jcpe.12854.

9. Ana P, Obradovic R, Ljiljana K, et al. Smoking and periodontal disease: a review. Med Biol 2007;14:53-59.

10. Darby IB, Hodge PJ, Riggio MP, et al. Clinical and microbiological effect of scaling and root planing in smoker and non-smoker chronic and aggressive periodontitis patients. J Clin Periodontol 2005;32(2):200206. DOI: 10.1111/j.1600-051X.2005.00644.x.

11. Farina R, Simonelli A, Rizzi A, et al. Effect of smoking status on pocket probing depth and bleeding on probing following non-surgical periodontal therapy. Minerva Stomatol 2010;59(1-2):1-12. English, Italian.

12. Chang J, Meng HW, Lalla E, et al. The impact of smoking on nonsurgical periodontal therapy: a systematic review and meta-analysis. J Clin Periodontol 2021;48(1):60-75. DOI: 10.1111/jcpe.13384.

13. César Neto JB, Rosa EF, Pannuti CM, et al. Smoking and periodontal tissues: a review. Braz Oral Res 2012;26(Suppl 1):25-31. DOI: 10.1590/ s1806-83242012000700005.

14. Johnson GK, Guthmiller JM. The impact of cigarette smoking on periodontal disease and treatment. Periodontol 2000 2007;44(1):178194. DOI: 10.1111/j.1600-0757.2007.00212.x.

15. Chatzopoulos GS, Mansky KC, Lunos S, et al. Sclerostin and WNT-5a gingival protein levels in chronic periodontitis and health. J Periodontal Res 2019;54(5):555-565. DOI: 10.1111/jre. 12659.

16. García-Martín A, Rozas-Moreno P, Reyes-García R, et al. Circulating levels of sclerostin are increased in patients with type 2 diabetes mellitus. J Clin Endocrinol Metab 2012;97(1):234-241. DOI: 10.1210/ jc.2011-2186.

17. Napimoga $\mathrm{MH}$, Nametala $\mathrm{C}$, da Silva FL, et al. Involvement of the Wnt- $\beta$-catenin signalling antagonists, sclerostin and dickkopf-related protein 1, in chronic periodontitis. J Clin Periodontol 2014;41(6):550557. DOI: $10.1111 /$ jcpe.12245.

18. Yakar N, Guncu GN, Akman AC, et al. Evaluation of gingival crevicular fluid and peri-implant crevicular fluid levels of sclerostin, TWEAK, RANKL and OPG. Cytokine 2019;113:433-439. DOI: 10.1016/j. cyto.2018.10.021. 